\title{
Segmentation of Fundus Eye Images Using Methods of Mathematical Morphology for Glaucoma Diagnosis
}

\author{
Katarzyna Stạpor ${ }^{1}$, Adam Świtonski ${ }^{1}$, Radim Chrastek $^{2}$, and \\ Georg Michelson ${ }^{3}$ \\ 1 Institute of Computer Science, Silesian University of Technology, Akademicka 16, \\ PL-44-100 Gliwice, Poland, \\ 2 Chair for Pattern Recognition, Friedrich-Alexander-University \\ Erlangen-Nuremberg, Martenstrasse 3, D-91058 Erlangen, Germany, \\ 3 Department of Ophthalmology, Friedrich-Alexander-University \\ Erlangen-Nuremberg Schwabachanlage 6, D-91054 Erlangen, Germany \\ delta@ivp.iinf.polsl.gliwice.pl
}

\begin{abstract}
In this paper the new method for automatic segmentation of cup and optic disc in fundus eye images taken from classical fundus camera is proposed. The proposed method is fully based on techniques from mathematical morphology. Detection of cup region makes use of watershed transformation with markers imposed, while optic disk is extracted based on geodesic reconstruction by dilation. The obtained results are encouraging.
\end{abstract}

\section{Introduction}

Glaucoma is a group of diseases characterized by the proceeding optic nerve neuropathy which leads to the rising diminution in vision field, ending with blindness. The correct optic disk (i.e. the exit of the optic nerve from the eye known as "blind spot") structure contains: neuroretinal rim of pink color and centrally placed yellowish cup [5]. The shape of the optic disc is more or less circular, interrupted by the outgoing vessels. Its size varies from patient to patient. Its diameter lies between 40 and 60 piksels on $640 \times 480$ color photographs. The cup is the area within the optic disc where no nerve fibers and blood vessels are present and in 3D image appears as an excavation. The neuroretinal rim is the area between optic disc border and cup border.

Glaucomatous changes in retina appearance embrace various changes in neuroretinal rim and cup, as the result of nerve fibers damages.

Optic disc structures evaluation is one of the most important examinations in glaucoma progress monitoring and diagnosis. Searching for glaucoma damages during routine examination is not an easy task and gives uncertain results even with the experienced ophthalmologist [5]. The existing methods of qualitative analysis are very subjective, while quantitative methods of optic disc morphology evaluation (cup to disc ratio, neuroretinal rim area) do not result in full 
diagnosis. The new, quantitative methods based on scanning-laser-tomography are expensive and accessible only in specialized ophthalmic centers.

Thus, there is a need for cheaper and more objective methods that enable automatic classification of digital fundus eye images (fei) into normal and glaucomatous ones. The first, but most important step in all such methods is automatic segmentation of optic disc structures from fei.

In the existing approaches to automatic segmentation of fei for supporting glaucoma examinations researchers focused on the detection of the optic disk. These methods make use of Hough transform [8], active contours [6], tools from mathematical morphology [12].

In [4] important proofs that shape of the eye cup and its numerical characteristics correlate with progress of glaucoma disease were presented.

As far as we know, no automatic method for the segmentation of cup from fei has been reported.

This paper presents the new method for automatic segmentation of cup region as well as optic disc from fei taken from classical fundus camera. The proposed method is fully based on techniques from mathematical morphology: mainly watershed transformation and geodesic reconstruction.

\section{Some Grey-Level Morphological Operators}

In this section we briefly define the basic morphological operators used in this paper (for a comprehensive presentation see [1,11]). Let $D_{f}$ and $D_{B}$ be subsets of $Z^{2}$ and $T=\left\{t_{\min }, \ldots, t_{\max }\right\}$ be an ordered set of gray levels. A grey-level image $f$ can be defined as a function:

$$
f: D_{f} \subset Z^{2} \rightarrow T
$$

Furthermore, we define another image known as a structuring element $B$ :

$$
B: D_{B} \subset Z^{2} \rightarrow T
$$

We will restrict to flat, symmetric structuring elements B. We can now write the four basic morphological operators: erosion, dilation, opening and closing as:

$$
\begin{gathered}
E^{B}(f)(x, y)=\inf _{(j, k) \in D_{B}}\{f(x-j, y-k)\}, \\
D^{B}(f)(x, y)=\sup _{(j, k) \in D_{B}}\{f(x-j, y-k)\}, \\
O^{B}(f)(x, y)=D^{B}\left(E^{B}(f)(x, y)\right), \\
C^{B}(f)(x, y)=E^{B}\left(D^{B}(f)(x, y)\right),
\end{gathered}
$$

Symmetric, morphological gradient of an image $f$ can be defined as:

$$
\operatorname{grad}(f)(x, y)=D^{B}(f)(x, y)-E^{B}(f)(x, y),
$$


Furthermore, we shall define geodesic transformation of an image $f$ : geodesic erosion and dilation of size $\mathrm{n}$ :

$$
\begin{aligned}
& E_{c(n)}^{B, g}(f)(x, y)=E_{c(1)}^{B, g}\left(\left(E_{c(n-1)}^{B, g}(f)\right)(x, y)\right. \\
& E_{c(1)}^{B, g}(f)(x, y)=\sup \left\{E^{B}(f)(x, y), g(x, y)\right\} \\
& D_{c(n)}^{B, g}(f)(x, y)=D_{c(1)}^{B, g}\left(\left(D_{c(n-1)}^{B, g}(f)\right)(x, y)\right. \\
& D_{c(1)}^{B, g}(f)(x, y)=\inf \left\{D^{B}(f)(x, y), g(x, y)\right\}
\end{aligned}
$$

reconstruction by dilation and reconstruction by erosion:

$$
\begin{aligned}
& R^{B, f}(g)(x, y)=D_{c(\infty)}^{B, g}(f)(x, y) \\
& R_{*}^{B, f}(g)(x, y)=E_{c(\infty)}^{B, g}(f)(x, y)
\end{aligned}
$$

where $D_{c(\infty)}^{B, g}\left(E_{c(\infty)}^{B, g}\right)$ is the limit which is obtained by iterating unit geodesic erosion of $f$ above (under) $g$ until stability is reached, i.e.:

$$
D_{c(i)}^{B, g}(f)(x, y)=D_{c(i+1)}^{B, g}(f)(x, y)
$$

Furthermore, we shall make use of the watershed transformation, for which we do not give a mathematical definition here.

\section{Detection of Cup Region Based on Watershed Transformation}

\subsection{The Color Space}

Having compared several color spaces, we found the contours of the cup region to appear most continuous and most contrasted against the background in the $a$ channel of the Lab color space [3] (image $f_{a^{-}}$Fig. 1a).

\subsection{Pre-processing}

First, we apply a shade-correction operator in order to remove slow background variations. Thus, we calculate:

$$
f_{a}^{1}=f_{a}-A S F\left(f_{a}\right)+k
$$

where $k$ is a positive constant and

$$
\left.A S F\left(f_{a}\right)=C^{n B}\left(\ldots\left(O^{2 B}\left(O^{B}\left(f_{a}\right)\right)\right)\right) \ldots\right),
$$

is the result of alternating sequential filtering of $f_{a}$ with $\mathrm{n}$ sufficiently large to remove the cup. This is the approximation of the slow variations of the background of image $f_{a}$. 
Next, the image $f_{a}^{1}$ is filtered in order to eliminate large grey level variations within a cup region caused by the outgoing vessels. We "fill" the vessels by applying a simple closing operation:

$$
f_{a}^{2}=C^{B}\left(f_{a}^{1}\right)
$$

with a structuring element $B$ bigger than the maximal width of vessels - we use a circle with a radius 15 . The result is shown in Fig. 1 b.

\subsection{Finding Markers for Watershed Transformation}

To automatically find the internal marker, we first localize approximately the cup region. As we know approximately the size of the cup, and assuming that parts of the cup region belong to the darkest parts of the image $f_{a}^{2}$, we apply a simple global thresholding:

$$
f_{a}^{3}=T_{t 1}\left(f_{a}^{2}\right)
$$

to obtain a binary image $f_{a}^{3}$. The parameter $t 1$ is chosen based on a cumulative histogram in a way that $2 \%$ of the darkest pixels will be considered as objects. The image $f_{a}^{3}$ contains cup region as well as other pathologies, like for example exudates. They are not very big, and they are far from reaching the size of the cup. Hence, we select the biggest particle of the image $f_{a}^{3}$ (giving image $f_{a}^{4}$ shown in Fig. 1c) which coincides with the candidate region containing the cup. The coordinates of the centroid $c$ of the candidate region in the image $f_{a}^{4}$ are calculated as:

$$
x_{c}=\frac{\sum_{x} \sum_{y} f_{a}^{4}(x, y) \cdot x}{\sum_{x} \sum_{y} f_{a}^{4}(x, y)}, y_{c}=\frac{\sum_{x} \sum_{y} f_{a}^{4}(x, y) \cdot y}{\sum_{x} \sum_{y} f_{a}^{4}(x, y)}
$$

The calculated centroid $c\left(x_{c}, y_{c}\right)$ is taken as the internal marker $m_{\text {int }}$ for watershed transformation. As external marker $m_{\text {ext }}$ we use a circle $C_{c u p}\left(c, r_{c u p}\right)$ with a center at the calculated centroid $c$ and a radius $r_{c u p}$ bigger than the diameter of the biggest cup (see Fig. 1d).

\subsection{Watershed Transformation}

In order to detect exact contours of the cup region, we apply the classical watershed transformation:

$$
f_{a}^{6}=W T^{m_{\text {int }} \cup m_{\text {ext }}}\left(f_{a}^{5}\right),
$$

to the morphological gradient of the filtered image $f_{a}^{2}$ :

$$
f_{a}^{5}=\operatorname{grad}\left(f_{a}^{2}\right)=D^{B}\left(f_{a}^{2}\right)-E^{B}\left(f_{a}^{2}\right)
$$

with internal $m_{\text {int }}$ and external $m_{\text {ext }}$ markers imposed to avoid oversegmentation of the image. The watershed transformation assigns to each local minimum of $f_{a}^{5}$ one catchment basin (one connected region), in a way that all $x \in D_{f_{a}^{5}}$ belong to a basin except a one pixel strong line that delimits the basins (the watershed line). This watershed line defines the exact contour of the cup. 


\subsection{Post-processing}

The shape irregularities in the segmentation result are due to the outgoing vessels or to low contrast. We can regularize the shape of the contour using standard morphological filtering techniques, i.e. smoothing by binary opening-closing operations of the resulted cup region. The final segmentation result is shown in Fig. 1f.

\section{Detection of the Optic Disc Using Geodesic Reconstruction}

Having compared several color spaces, we found that the contours of the optic disc are most contrasted against the background in the $G$ channel of the RGB color space (Fig. 2a - image $f_{G}$ ). Finding the contours of the optic disc is based on the morphological reconstruction by dilation of the image $f_{G}$ :

$$
f_{G}^{1}=R_{D}^{B, g}\left(f_{G}\right)
$$

starting from a properly constructed marker image $g(x)$ (Fig. 2b)

$$
g(x)=\left\{\begin{array}{lll}
0 & \text { if } & x \in C_{\text {disc }}\left(c, r_{\text {disc }}\right) \\
f_{G} & \text { if } & x \notin C_{\text {disc }}\left(c, r_{\text {disc }}\right)
\end{array}\right.
$$

where $C_{d i s c}\left(c, r_{d i s c}\right)$ is a circle with a center, being the centroid $c\left(x_{c}, y_{c}\right)$ calculated during cup region detection (as described in the previous section), and a radius $r_{\text {disc }}$ equal to a radius of a typical optic disc. This circle is a candidate region comprising the optic disc inside. All the pixels in a marker image $g(x)$ which are comprised in a circle $C_{d i s c}\left(c, r_{d i s c}\right)$ are set to zero (means black). The reconstruction operator propagates the values of $f_{G}(x)$ of pixels $x$ next to the circle into the circle by the successive geodesic dilation under the mask $f_{G}$. As optic disc is entirely comprised within the circle, it is completely removed, whereas regions that are not entirely comprised in the circle are nearly entirely reconstructed. Next, a simple thresholding operation is applied to the difference between the original image $f_{G}$ and the reconstructed image $f_{G}^{1}$ :

$$
f_{G}^{2}=T_{t 2}\left(f_{G}-f_{G}^{1}\right),
$$

The threshold $T_{t 2}$ has been experimentally set so as to differentiate between optic disc region and the rest of the image. To avoid influence of the vessels in the image $f_{G}^{2}$ (Fig. 2e), the binary closing operation is performed on the image $f_{G}^{2}$ which gives the result - the optic disc region. Its contour is shown in Fig. $2 \mathrm{f}$ imposed on the input image. 


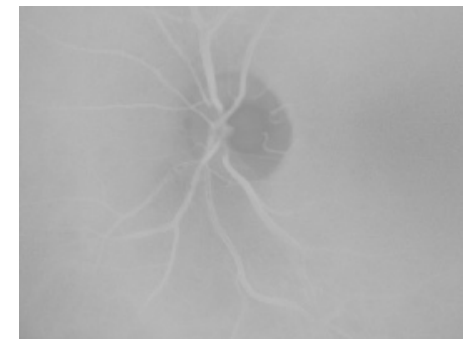

a)

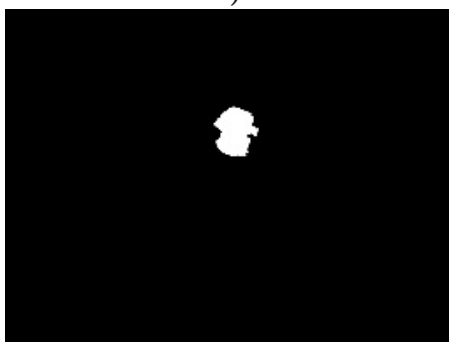

c)

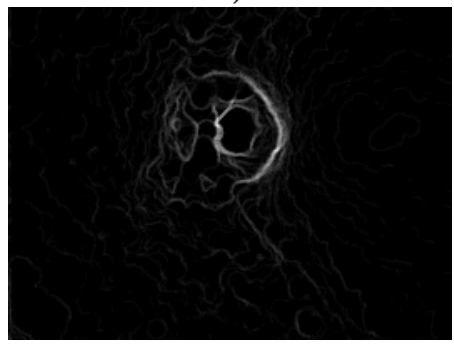

e)

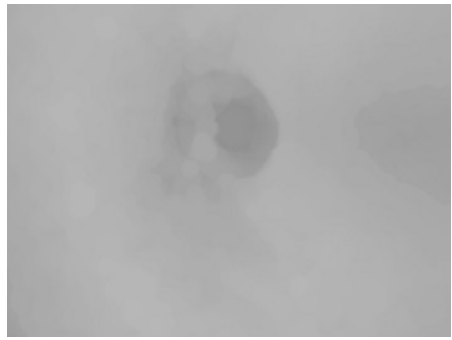

b)

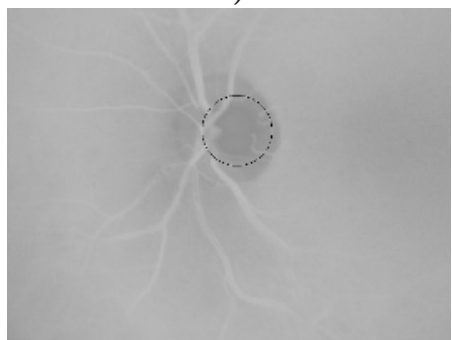

d)

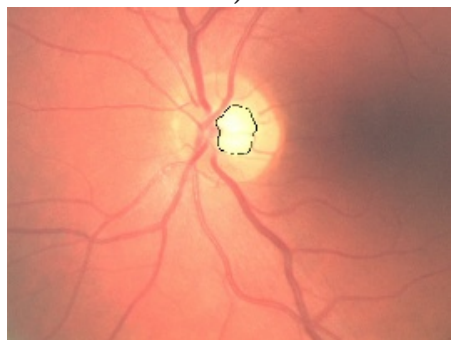

f)

Fig. 1. The detection of the cup region: a) channel $a$ of the input image (image $f_{a}$ ); b) channel $a$ after closing operation (image $f_{a}^{2}$ ); c) candidate region on the thresholded image (image $f_{a}^{4}$ ); d) channel $a$ with the external marker imposed; e) morphological gradient of image $f_{a}^{2}$ (image $f_{a}^{5}$ ); f) result of the segmentation - watershed line imposed on the input image

\section{Results and Conclusions}

We tested the algorithm on 50-ty images of size $640 \times 480$ that have not been used for the development of the algorithm. The images were taken from the Canon CF-60Uvi fundus-camera. In 48 images we could found exact cup and disc contours. However in two images the contrast was to low, the algorithm failed and the result was not acceptable. It is important to note that the contours of the cup and optic disc obtained as a result of the presented segmentation methods coincide with the contours marked by an ophthalmologist. The results of using the presented method are very encouraging. 


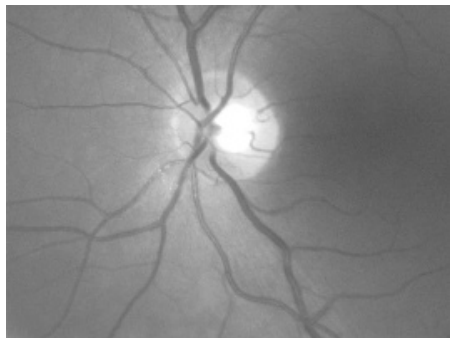

a)

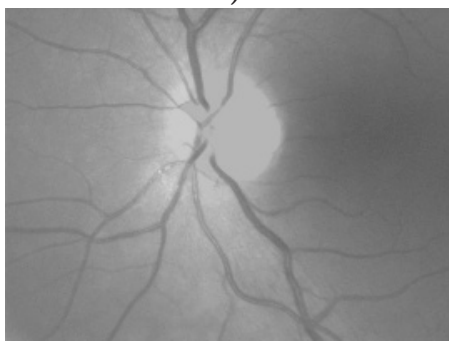

c)

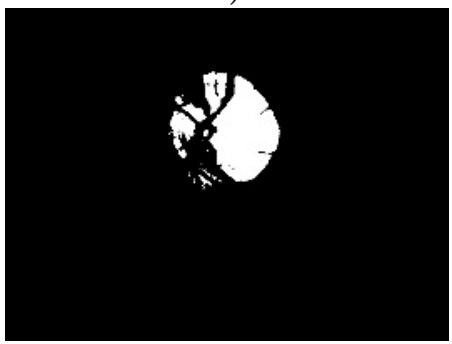

e)

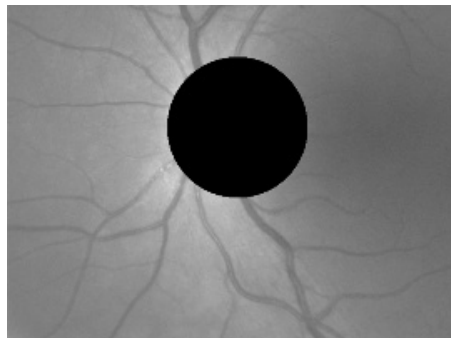

b)

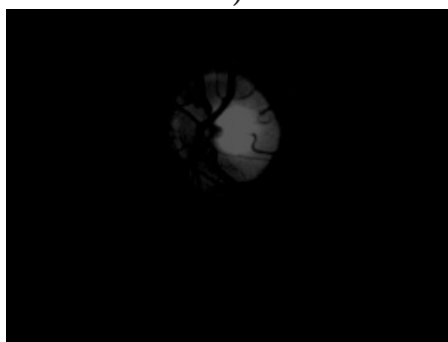

d)

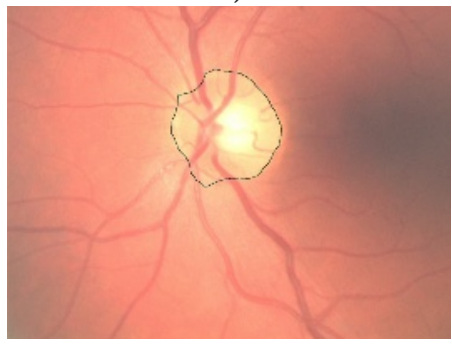

f)

Fig. 2. The detection of the optic disc: a) channel $\mathrm{G}$ of the input image (image $f_{G}$ ); b) the marker image $g(x)$; c) the reconstructed image $f_{G}^{1}$; d) the difference between original and reconstructed images; e) the result after thresholding the difference image; f) result of the segmentation - contours of the detected optic disc imposed on the input image

\section{References}

1. Beucher S., Meyer F.: The morphological approach to image segmentation: the watershed transformation. E.R. Dougherty (Eds.): Mathematical morphology in image processing (1993) 433-481

2. Goh K.G, et al: ADRIS: an Automatic Diabetic Retinal Image Screening system. K. J. Cios (Eds.): Medical Data Mining and Knowledge Discovery. Springer-Verlag New York (2000) (181-210)

3. Gonzalez R.C., Woods R.E.: Digital image processing. Prentice-Hall (2002)

4. Jonas J. et al.: Ophthalmoscopic evalutation of the optic nerve head. Survey of Ophthalmology. Vol. 43, No. 4 (January - February 1999) 
5. Kanski J. et al. Glaucoma: a color manual of diagnosis and treatment. ButterworthHeinemann (1996)

6. Morris D.T., Donnison C.: Identifying the Neuroretinal Rim Boundary Using Dynamic Contours. Image and Vision Computing, Vol. 17. (1999) 169-174

7. Osareh A., et al.: Classification and localisation of diabetic related eye disease. A. Heyden et al. (Eds.): ECCV 2002, LNCS 2353 (2002) 502-516

8. Pinz A., et al.: Mapping the human retina. IEEE Trans. Medical Imaging, Vol.1, (1998) (210-215)

9. soi Soille P.: Morphological Image analysis: principles and applications. SpringerVerlag, Berlin (1999)

10. Tamura S., Okamoto Y.: Zero-crossing interval correction in tracing eye-fundus blood vessels. Pattern Recognition, Vol.21, No. 3, (1988) (227-233)

11. Vincent L.: Morphological grayscale reconstruction in image analysis: applications and efficient algorithms. IEEE Trans. On Image processing, Vol.2, No. 2, (1993), (176-201)

12. Walter T., Klein J.: Segmentation of color fundus images of the human retina: detection of the optic disc and the vascular tree using morphological techniques. Proc. 2nd Int. Symp. Medical Data Analysis, (1999) (282-287) 